Pacific Journal of Mathematics

KRULL-SCHMIDT AND CANCELLATION OVER LOCAL 


\title{
KRULL-SCHMIDT AND CANCELLATION OVER LOCAL RINGS
}

E. Graham Evans, JR.

\begin{abstract}
This paper proves a partial converse to the Krull-Schmidt theorem for Hensel local rings and a cancellation result for modules in terms of the endomorphism ring of the module. The second result is then used to prove the cancellation theorem for finitely generated modules over local rings.
\end{abstract}

All rings in this paper have units. A module $M$ is indecomposable if $M \cong A \oplus B$ implies $A=0$ or $B=0$. A ring $R$ satisfies the Krull-Schmidt Theorem if every finitely generated $R$ module is uniquely a direct sum of indecomposable modules. A module $M$ can be cancelled if $M \oplus A$ is isomorphic to $M \oplus B$ implies $A$ is isomorphic to $B$. Usually one only considers cancellation when all the modules $M, A$, and $B$ are finitely generated although this paper does not need these hypotheses. Local ring includes the noetherian property while quasi-local is used for a ring with only one maximal ideal.

This investigation began when the author noticed that Swan's proof of the Krull-Schmidt Theorem for complete local rings passes unchanged to Hensel local rings. Swan kindly supplied the author with an example of failure of Krull-Schmidt which generalizes to all not Hensel local rings. Finally we prove that the type of failure of Krull-Schmidt given by Swan's example is the only type possible by proving a cancellation theorem strong enough to prove that any finitely generated module over a local ring can be cancelled. I wish to thank Professors Artin, Kaplansky, and Swan whose lectures and discussions introduced me to much of this material.

The outline of this paper is as follows: Section 1 presents Swan's example of the failure of Krull-Schmidt, $\S 2$ shows how for a given not Hensel local ring $R$ we can find a local ring $T$ which is a finite $R$ algebra for which the Krull-\$chmidt Theorem fails, and $\S 3$ examines cancellation properties of modules in terms of their endomorphism rings.

1. Example of R.G. Swan. Let $R$ be a local domain which is not a field. Assume $R / \mathrm{m}=k$ has characteristic not equal to 2 . Let $A=\left(R[x, y] /\left(x^{3}-x^{2}+y^{2}\right)\right)_{(x, y)}$. Then the Krull-Schmidt theorem fails for $A$. Let $z=y / x$ in the quotient field of $A$ and let $B=A[z]$. Then $B$ has just 2 maximal ideals, $M_{1}=(M, z-1)$ and $M_{2}=(M, z+1)$ where $M$ is the maximal ideal of $A$. Since $M_{1}+M_{2}=B$ we have an exact sequence of $B$ modules 


$$
0 \rightarrow M_{1} \cap M_{2} \rightarrow M_{1} \oplus M_{2} \rightarrow B \rightarrow 0
$$

which splits over $B$. Therefore, $M_{1} \oplus M_{2} \cong B \oplus\left(M_{1} \cap M_{2}\right)$ over $B$ and hence over $A$. All four modules here are torsion free rank 1 over $A$ and so are indecomposable. If $B \cong M_{1}$ over $A$, then $B \cong M_{1}$ over $B$ since $B$ and $M_{1}$ are torsion free rank 1 modules and $A$ and $B$ have the same quotient field (any isomorphism would look like multiplication by an element of the quotient field). But this is impossible since $B_{M_{i}}$ is local of dimension $\geqq 2$ so $M_{i}$ needs at least 2 generators as a $B$ module.

2. Swan's example generalized. To begin this section we first need some definitions. A quasi-local ring $R$ with unique maximal ideal $\mathfrak{m}$ is called Hensel if for every monic polynomial $f(x)$ over $R$ such that $f(x) \equiv g_{0}(x) h_{0}(x) \bmod \mathfrak{m} R[x]$ where $g_{0}(x)$ and $h_{0}(x)$ are monic and such that $g_{0} R[x]+h_{0} R[x]+\mathfrak{m} R[x]=R[x]$, then there exist monic polynomials $g(x)$ and $h(x)$ such that $f(x)=g(x) h(x), g(x) \equiv g_{0}(x) \bmod \mathfrak{m} R[x]$ and $h(x) \equiv h_{0}(x) \bmod m R[x]$. This is equivalent to being able to lift idempotents from $A / \mathfrak{m} A$ to $A$ for all finite $R$ algebras $A$. The definition and basic properties are due to Azumaya [1]. Note that Azumaya allowed (and in fact required) $A$ to be noncommutative. Several current treatments only consider commutative $A$ 's. This does not change the class of Hensel rings but does deaden the ability to see noncommutative applications such as the one in this section.

The integers localized at (5) is a local ring which is not Hensel for $x^{2}+1 \equiv(x+2)(x+3) \bmod 5$ is a factorization which cannot be lifted. But the integers localized at (5) satisfies the Krull-Schmidt theorem since it is a principal ideal domain. The following theorem shows that there is a strong connection between Hensel and KrullSchmidt properties of local rings.

Theorem 1. Let $R$ be a local ring. Then every $R^{\prime}$ which is a local ring and a finite $R$ algebra satisfies the Krull-Schmidt theorem if and only if $R$ is Hensel.

Proof. If $R$ is Hensel, then any commutative ring which is a finite $R$ algebra is a direct sum of Hensel rings (see, for example [7, 43.1 and 43.16]). Hence for one half we only need to prove that Hensel rings satisfy Krull-Schmidt. Swan's proof of Krull-Schmidt for complete local rings $R$ [10, Remark on page 566] only needs the ability to lift idempotents in finite algebras $A$ over $B$ from $A / \mathrm{m} A$ to $A$. But this ability characterizes Hensel rings. For more details in Hensel case see [9].

To prove the other half we need to mimic Swan's example of 
failure of Krull-Schmidt. We first reduce to the case of domains.

Lemma 1. If $R$ is a local ring which is not Hensel, then there exists a domain image of $R$ which is not Hensel.

Proof. $R$ is Hensel if and only if $R_{\text {red }}=R /$ nilpotent is Hensel. [8, page 5]. So we can assume $R$ is reduced. Then $0=P_{1} \cap \cdots \cap P_{n}$ with the $P_{i}$ prime. Hence to prove the lemma it is enough to prove the following:

LeMma 2. If $R$ is a local ring with ideals $\mathfrak{a}$ and $\mathfrak{b}$ such that $\mathfrak{a} \cap \mathfrak{b}=0$ such that $R / \mathfrak{a}$ and $R / \mathfrak{b}$ are Hensel, then $R$ is Hensel.

Proof. Following Nagata's description of Henselization [7, pp. 179-188] we pick $T$ an integrally closed domain with only one maximal ideal $M$ such that $T$ maps onto $R$ with Kernel $K$. Let $A$ and $B$ be the complete inverse images of $a$ and $\mathfrak{b}$. Let $T^{\prime}$ be the integral closure of $T$ in the algebraic closure $L$ of the quotient field of $T, H$. Let $M^{\prime}$ be some maximal ideal of $T$. Let $G$ be the elements of the Galois group of $L$ over $H$ which send $M^{\prime}$ to itself. Let $T^{\prime \prime}$ be the fixed ring of $G$. Then $\widetilde{R}$, the Henselization of $R$, is $T^{\prime \prime}{ }_{\left(M^{\prime} \cap T^{\prime \prime}\right)} / K T^{\prime \prime}{ }_{\left(M^{\prime} \cap T^{\prime \prime}\right)}$ • But since $R / \mathfrak{a}$ and $R / \mathfrak{b}$ are Hensel we have $R / a=T^{\prime \prime}{ }_{\left(M^{\prime} \cap T^{\prime \prime}\right)}$ and $R / \mathfrak{b}=T^{\prime \prime}{ }_{\left({ }^{\prime} \cap T^{\prime \prime}\right)} / B T^{\prime \prime}{ }_{\left(H^{\prime} \cap T^{\prime \prime}\right)}$. But since $T^{\prime \prime}{ }_{\left(M^{\prime} \cap T\right)}$ is faithfully flat over $T$ we have

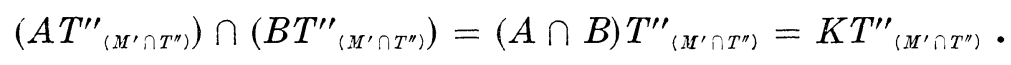

Hence $\widetilde{R}$, is a submodule of $R / \mathfrak{a} \oplus R / \mathfrak{b}$. Thus $\widetilde{R}$ is finitely generated. But if $\mathfrak{m}$ is the maximal ideal of $R$, then $\widetilde{R} / \mathfrak{m} \widetilde{R} \cong R / \mathfrak{m}[7,43.3]$. Hence, by Nakayama's lemma $\widetilde{R}$ is cyclic and hence equals $R$ and $R$ is Hensel as desired.

Hence to prove our result we can pass to $R$ being a local domain which is not Hensel. Now we can apply Nagata's criteria that a quasi-local domain is Hensel if and only if every domain integral over it is quasi-local. [7, 43.12]. Hence we can find a domain $R^{\prime}$ integral over $R$ which has at least two maximal ideals $M_{1}$ and $M_{2}$.

By picking $x_{1} \in M_{1}-M_{2}$ and $x_{2} \in M_{2}-M_{1}$ and passing to $R^{\prime}=$ $R\left[x_{1}, x_{2}\right]$ we can assume $R^{\prime}$ is finitely generated as an $R$ module. Let $A$ equal the intersection of the maximal ideals of $R^{\prime}$. Then $A$ is a finitely generated $R$ module and so is $R[A]$. But $R[A]$ is local.

Except for minor pathology $R[A]$ and $R^{\prime}$ can be used as the $A$ and $B$ in Swan's example (respectively). That is, if $M_{1}$ and $M_{2}$ both needed 2 generators as $R^{\prime}$ modules we would be done. This would be true if the dimension of $R_{M_{i}}^{\prime}$ were at least 2 . 
In general we pass to $S=R[x] /\left(x^{2}\right)$ and $T=R^{\prime}[x] /\left(x^{2}\right)$ and look at the maximal ideals $\left(M_{1}, x\right)=M_{1}^{\prime}$ and $\left(M_{2}, x\right)=M_{2}^{\prime}$. Then

$$
0 \rightarrow M_{1}^{\prime} \cap M_{2}^{\prime} \rightarrow M_{1}^{\prime} \oplus M_{2}^{\prime} \rightarrow T \rightarrow 0
$$

is exact and split. The modules are isomorphic over $T$ if and only if they are isomorphic over $S$ since it is enough to know what an isomorphism does on the part annihilated by $x$ to know what it does on everything.

Finally we check that $M_{1}^{\prime}$ are not principal over $T$.

Lemma 3. If $R$ is a domain and $M$ is a proper ideal of $R$, then the ideal generated by $M$ and $x$ in $R[x] /\left(x^{2}\right)$ is not principal.

Proof. Pick $m \in M-\{0\}$. Let $a x+b \in R[x] /\left(x^{2}\right)$ generate $(M, a)$. Then there exist $r_{i} \in R$ such that

(1) $\left(r_{1} x+r_{2}\right)(a x+b)=m$ and

(2) $\left(r_{3} x+r_{4}\right)(a x+b)=x$

Expanding (1) we get

(i) $r_{1} b+r_{2} a=0$ and

(ii) $r_{2} b=m$.

Expanding (2) we get

(iii) $r_{2} b=0$

(iv) $r_{3} b+r_{4} a=1$

From (iii) we get $r_{4}=0$ or $b=0$. But if $b=0$ then the ideal (ax) could not contain $m \neq 0$ by (ii). Hence $r_{4}=0$. Then from (iv) we get $r_{3} b=1$. But then $b=\left(1-r_{3} a x\right)(a x+b)$. Hence the ideal $(a x+b)$ contains $b$ which is a unit whereas $(M, x)$ is clearly proper.

Hence $(M, x)$ is not principal.

REMARK. This is a simplification of a result suggested to me by Swan. His result which is proved by the same methods is that if an ideal $(M, x)$ in $R[x]$ is principal then $M$ is generated by an idempotent. On the other hand if $M$ is generated by an idempotent $e$ then $(M, x)=(e+(1-e) x)$ as an ideal in $R[x]$.

This completes the proof of Theorem 1. One might ask for stronger results of this type. In particular you could ask if the dimension of $R$ were big enough and $R$ not Hensel then the Krull-Schmidt theorem fails for $R$ itself. A difficulty involved is that $R$ could be very close to being Hensel. Say, for example, if $R=k\left[\left[x_{1}, x_{2}, \cdots, x_{n}\right]\right][y]_{\left(x_{1}, \cdots x_{n}, y\right)}$.

3. The failure of Krull-Schmidt in the above section was always of the type $A \oplus B \cong C \oplus D$ all distinct and indecomposable. On the other hand we have 
Proposition 1. Let $R$ be a local ring and let $A, B$, and $C$ be finitely generated $R$ modules such that $A \oplus B$ is isomorphic to $A \oplus C$, then $B$ is isomorphic to $C$.

Proof. After completing and applying the Krull-Schmidt theorem over $\hat{R}$ we can conclude that $\hat{B}$ is isomorphic to $\hat{C}$. But then a theorem of Grothendeick [5, 2.5.8] asserts that $B$ is isomorphic to $C$. The details are carried out in Vasconcelos's paper [12].

Of course, the above proof makes rather heavy use of finite generation of $A, B$, and $C$. For example $\hat{R}$ and $R$ have isomorphic completions but are not isomorphic unless $R$ is complete.

The next theorem strengthens the above cancellation result following ideas of Bass [2] and Dress [3].

If $A$ is the endomorphism ring of a finitely generated module over a local ring, then $A$ is itself finitely generated over $R$ (since $R$ is noetherian). Hence a theorem of Bass [2, Corollary 6.5] assures us that 1 is in the stable range for $A$. That is, if $a$ and $b$ are elements of $A$ such that $A a+A b=A$, then there exists a $t \in A$ such that $a+t b$ is a unit of $A$.

The following theorem is a natural generalization of a remark of Kaplansky that if 1 is in the stable range of $R$ and $R \oplus A$ is isomorphic to $R \oplus B$, then $A$ and $B$ are isomorphic. See also Swan [11, Proposition 11.7] for an earlier result of this type.

THEOREm 2. Let $R$ be any ring and $A, B$, and $C$ any modules such that $A \oplus B$ is isomorphic to $A \oplus C$ and such that the endomorphism ring, $T$, of $A$ has 1 in the stable range, then $B$ is isomorphic to $C$.

Proof. Let $f_{M, N}$ denote an $R$ homomorphism from $N$ to $M$.

We are presumed to have maps $f_{A \oplus C, A \oplus B}$ and $g_{A \oplus B, A \nsubseteq C}$ whose composition in each direction are the identities. Thinking of $f$ and $g$ as $2 \times 2$ matrices we have

$$
\left(\begin{array}{ll}
g_{A, A} & g_{A, C} \\
g_{B, A} & g_{B, C}
\end{array}\right)\left(\begin{array}{ll}
f_{A, A} & f_{A, B} \\
f_{C, A} & f_{C, B}
\end{array}\right)=\left(\begin{array}{ll}
I_{A} & 0 \\
0 & I_{B}
\end{array}\right)
$$

where $I_{M}$ is the identity on $M$. Multiplying out the left hand side we get

$$
\left(\begin{array}{ll}
g_{A, A} f_{A, A}+g_{A, C} f_{C, A} & g_{A, A} f_{A, B}+g_{A, C} f_{C, B} \\
g_{B, A} f_{A, A}+g_{B, C} f_{C, A} & g_{B, A} f_{A, B}+g_{B, C} f_{C, B}
\end{array}\right)=\left(\begin{array}{ll}
I_{A} & 0 \\
0 & I_{B}
\end{array}\right) .
$$

Hence $T f_{A, A}+T g_{A, C} f_{C, A}=T$. Hence there exist $t \in T$ with $f_{A, A}+$ $t g_{A, C} f_{C, A}=u$ a unit.

Hence if we use the map 


$$
g^{\prime}=\left(\begin{array}{ll}
I_{A} & t g_{A, C} \\
g_{B, A} & g_{B, C}
\end{array}\right)
$$

in place of $g$ we get

$$
g^{\prime} f=\left(\begin{array}{ll}
u & v_{A, B} \\
0 & I_{B}
\end{array}\right)
$$

$g^{\prime} f$ is clearly an isomorphism since we can make $u$ be the identity by multiplying by $u^{-1}$ and then use elementary row operations to remove what is left over. But $g^{\prime} f$ an isomorphism and $f$ an isomorphism implies $g^{\prime}$ is. But then

$$
g^{\prime \prime}=\left(\begin{array}{cc}
g_{B, A} & I_{B} \\
-I_{A} & 0
\end{array}\right)\left(\begin{array}{ll}
I_{A} & t g_{A, C} \\
g_{B, A} & g_{B, C}
\end{array}\right)\left(\begin{array}{cc}
I_{A} & -t g_{A, C} \\
0 & I_{C}
\end{array}\right)
$$

is an isomorphism.

$$
g^{\prime \prime}=\left(\begin{array}{ll}
I_{A} & 0 \\
0 & g_{B, C}-g_{B, A} t g_{A, C}
\end{array}\right)
$$

and hence $g_{B, C}-g_{B, A} t g_{A, C}$ is an isomorphism from $C$ to $B$.

Corollary 1. Let $R$ be a local ring, $A$ a finitely generated $R$ module, and $B$ and $C$ any $R$ modules such that $A \oplus B$ is isomorphic to $A \oplus C$. Then $B$ is isomorphic to $C$.

Proof. Immediate from Theorem 2 and the proceeding remarks.

REMARKS. We note that Theorem 2 applies even for non-noetherian rings. Estes and $\mathrm{Ohm}$ in [4] give examples of commutative rings $R$ of any finite Krull dimension with 1 in the stable range. Heinzer in [6] gives such examples where the maximal spectrum is a noetherian space. Since any commutative ring equals its endomorphism ring, Theorem 2 shows that these rings are cancellable from any modules over them. I conjecture that these examples behave like the examples in Theorem 1 of discrete valuation rings which were not Hensel. More specifically, the conjecture is if $R$ is ring with noetherian maximal spectrum such that every finite $R$ algebra, $T$, has $d+1$ in the stable range, then the dimension of the maximal spectrum is less than or equal to $d$.

\section{REFERENCES}

1. G. Azumaya, On maximally central algebras, Nagoya Math. J., 2 (1950), 119-150.

2. H. Bass, K-theory and stable algebra, Publ. Math. I.H.E.S., no. 22, Paris (1964). 
3. A. Dress, On the decomposition of modules, Bull. Amer. Math. Soc., 75 (1969), 984986.

4. D. Estes and J. Ohm, Stable range in commutative rings, J. Algebra, 7 (1967), 343-362.

5. A. Grothendieck, Elemente de geometrie algebrique, Chapitre IV, Partie 1, Publ. Math. I.H.E.S., no 24, Paris (1965).

6. W. Heinzer, J-noetherian integral domains with 1 in the stable range, Proc. Amer. Math. Soc., 19 (1968), 1369-1372.

7. M. Nagata, Local Rings, Interscience, New York, 1962.

8. M. Raynaud, Anneaux Loceaux Henseliens, Lecture Notes in Mathematics 169, Springer-Verlag, Berlin (1970).

9. A. Simis, On the Krull-Schmidt Theorem for Orders over Hensel Rings and Valuation Rings, Queen's Mathematical Preprints No. 1971-18. Kingston (1971).

10. R. G. Swan, Induced representations and projective modules, Annals of Math., $7 \mathbf{1}$ (1960), 552-578.

11. - Algebraic K-Theory, Lecture Notes in Mathematics 76, Springer-Verlag, Berlin (1969).

12. W. Vasconcelos, On local and stable cancellation, An. Acad. Brasil Ci.. 37 (1965) $389-393$.

Received February 23, 1972. This research was partially supported by NSF Grant GP22927.

Massachusetts Institute of Technology

Current address: University of Illinois at Urbana-Champaign 



\section{PACIFIC JOURNAL OF MATHEMATICS}

\section{EDITORS}

D. Gilbarg and J. Milgram Stanford University

Stanford, California 94305

R. A. Beaumont

University of Washington

Seattle, Washington 98105
J. DugundJI

Department of Mathematics

University of Southern California

Los Angeles, California 90007

RICHARD ARENS

University of California

Los Angeles, California 90024

ASSOCIATE EDITORS
E. F. BECKENBACH
B. H. NeumanN
F. WOLF
K. YoSHIDA

\section{SUPPORTING INSTITUTIONS}

UNIVERSITY OF BRITISH COLUMBIA

CALIFORNIA INSTITUTE OF TECHNOLOGY

UNIVERSITY OF CALIFORNIA

MONTANA STATE UNIVERSITY

UNIVERSITY OF NEVADA

NEW MEXICO STATE UNIVERSITY

OREGON STATE UNIVERSITY

UNIVERSITY OF OREGON

OSAKA UNIVERSITY
UNIVERSITY OF SOUTHERN CALIFORNIA

STANFORD UNIVERSITY

UNIVERSITY OF TOKYO

UNIVERSITY OF UTAH

WASHINGTON STATE UNIVERSITY

UNIVERSITY OF WASHINGTON

$\stackrel{*}{*} \stackrel{*}{*} \stackrel{*}{*}$ AMERICAN MATHEMATICAL SOCIETY

NAVAL WEAPONS CENTER 


\section{Pacific Journal of Mathematics}

\section{Vol. 46, No. $1 \quad$ November, 1973}

Allan Francis Abrahamse, Uniform integrability of derivatives on $\sigma$-lattices .......................................... 1

Ronald Alter and K. K. Kubota, The diophantine equation $x^{2}+D=p^{n} \ldots \ldots \quad 11$

Grahame Bennett, Some inclusion theorems for sequence spaces .......... 17

William Cutler, On extending isotopies ........................ 31

Robert Jay Daverman, Factored codimension one cells in Euclidean

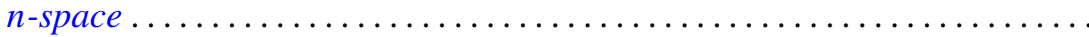

Patrick Barry Eberlein and Barrett O’Neill, Visibility manifolds ............ 45

M. Edelstein, Concerning dentability .......................... 111

Edward Graham Evans, Jr., Krull-Schmidt and cancellation over local

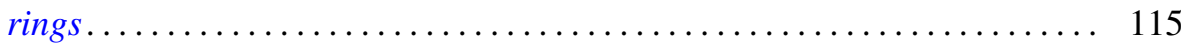

C. D. Feustel, A generalization of Kneser's conjecture ................ 123

Avner Friedman, Uniqueness for the Cauchy problem for degenerate parabolic equations .......................................... 131

David Golber, The cohomological description of a torus action ............ 149

Alain Goullet de Rugy, Un théorème du genre "Andô-Edwards" pour les

Fréchet ordonnés normaux..............................

Louise Hay, The class of recursively enumerable subsets of a recursively enumerable set ........................................

John Paul Helm, Albert Ronald da Silva Meyer and Paul Ruel Young, On orders of translations and enumerations...

Julien O. Hennefeld, A decomposition for $B(X)^{*}$ and unique Hahn-Banach

extensions

Gordon G. Johnson, Moment sequences in Hilbert space .

Thomas Rollin Kramer, A note on countably subparacompact spaces ...

Yves A. Lequain, Differential simplicity and extensions of a derivation ....

Peter Lorimer, A property of the groups Aut $\mathrm{PU}\left(3, q^{2}\right) \ldots$

225

Yasou Matsugu, The Levi problem for a product manifold.

231

John M.F. O'Connell, Real parts of uniform algebras ......

William Lindall Paschke, A factorable Banach algebra without bounded

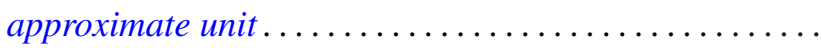

Ronald Joel Rudman, On the fundamental unit of a purely cubic field ....

Tsuan Wu Ting, Torsional rigidities in the elastic-plastic torsion of simply connected cylindrical bars .........................

Philip C. Tonne, Matrix representations for linear transformations on analytic sequences...................................

Jung-Hsien Tsai, On E-compact spaces and generalizations of perfect

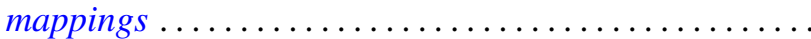

Alfons Van Daele, The upper envelope of invariant functionals majorized by an invariant weight. .. 